\section{Business skills for postdocs}

The Keck Graduate Institute in Claremont, California, has established the first US

professional master's programme offering business and industry training specifically to postdoctoral fellows. The programme builds on the Professional Science Master's degree, an increasingly popular business-skill building option offered to graduate students.

The institute's president, Sheldon Schuster (pictured), says that the programme aims to respond to industry complaints that academic postdocs often don't understand the corporate culture. A pilot programme will begin this September with four or five students taking existing business courses at Keck. By January 2010, Schuster expects up to two dozen openings as the institution customizes the programme to meet postdoc-specific needs, such as developing industry projects that tackle more complicated scientific questions than graduate students address.

The institute was the first to offer a degree aimed at producing PhD students with business skills. Now there are more than 120 such programmes across the United States.

Many in industry are eager to couple the postdoc's scientific sophistication with business savvy. "For technical companies like us, there is a lot of value in educating people who have demonstrated strong scientific depth with some business skills," says Jim Widergren, corporate vice-president of Asia Pacific and Latin American operations at
Beckman Coulter, a diagnostic biotechnology company based in Fullerton, California.

Joseph Panetta, president and chief executive of BIOCOM, a biotech industry organization based in San Diego, California, says that BIOCOM created a two- to three-month programme - dubbed the Life Sciences Immersion Program - last year to offer industry skill-building and networking opportunities to postdocs. "Our idea is to give postdocs a bridge into the world of industry," he says. The economic downturn put the programme on hold, but Panetta hopes to launch it within the next year. Some postdocs are likely to scoff at yet more training, but those having difficulty securing an academic post may appreciate a new opportunity. "There is going to be a very targeted population of postdoctoral scholars for which Keck's focused industry programme will be a welcome option," says Cathee Johnson Phillips, executive director of the National Postdoctoral Association in Washington DC. She says that there is a growing trend among postdoctoral students to acquire the skills necessary to compete for jobs outside of academia.

Schuster emphasizes the importance of exposing postdocs to all the career options available, especially given the paucity of academic posts compared to the large number of postdocs. "I would be shocked if more professional master programmes specific to postdocs don't pop up in the future," he says. Virginia Gewin

\section{POSTDOC JOURNAL}

\section{Finding the perfect match}

Applying for a faculty job is surprisingly analogous to dating. I screen job adverts and respond to those that look like good matches. If a search committee decides that I'm an attractive candidate, it will suggest that we meet face-toface to get to know each other. As I prepare my interview outfit and my research talk, I wonder when I'll no longer have to apply to search e-mail me back. I daydream that I'll soon be out of the job market and in a committed relationship with a department that appreciates my research committees that never call or and doesn't have unrealistic teaching expectations.

During my interview I build a rapport with potential colleagues as we share our research interests and future aspirations for the department. We explore whether we might become better scientists by working together. Our discussion might become more intimate: how do I feel about collaboration? How many graduate students would I like to work with?

Light-headed, I return home and wait for news. I restrain myself from serially checking e-mail or from hovering by the phone. Time passes and I wonder why the search committee hasn't called. Perhaps it is busy, or on holiday, or maybe its mother is ill. Would it respond well to a music mix tape? Maybe it's just not that into me. As a distraction, I throw myself into other job applications. Someday soon I'm sure I'II meet that perfect search committee - and we'll know for certain that we are meant to be together.

Julia Boughner is a postdoc in evolutionary developmental biology at the University of Calgary, Canada.
IN BRIEF

\section{Foreign admissions fall}

Offers to prospective international students by US graduate schools have fallen by $3 \%$ since last year, the first decline since 2004, according to a report by the Council of Graduate Schools in Washington DC.

More than half of the roughly 250 institutions that responded to the council's survey reported offering fewer places to international students. The countries most affected were India and South Korea, which fell by $16 \%$ each. China bucked the trend with a rise of $13 \%$.

Nathan Bell, the council's director of research and policy analysis, attributes the declines to a reduction in recruiting efforts abroad because of the recession.

\section{Data manager for Europe}

The European Molecular Biology Laboratory's European Bioinformatics Institute, based in Cambridge, UK, is expanding its data storage and handling capacity to become the hub of the European Life Sciences Infrastructure for Biological Information (ELIXIR) initiative.

ELIXIR is funded by the European Commission and individual federal governments, non-profit organizations and agencies across Europe and aims to create a way to manage and store data from the life sciences and information from thousands of labs. Its establishment could create new positions in bioinformatics and information technology.

\section{IT sector takes a hit}

The global recession has hit the information and communications technology industry hard. But research and development in the sector is performing solidly, according to a report issued last month by the Organisation for Economic Co-operation and Development. The organization, which collects economic and science-policy information, among other responsibilities, represents 30 nations worldwide.

The report found that venture-capital investment in the sector has markedly slowed since mid-2008. But it continues to flow into clean technologies in the sector, an area that should continue to receive a major share of venture capital, the document predicts. 